\title{
Sorgende Freunde
}

\section{Nichtverwandschaftliche Beziehungen als Familienersatz}

\author{
Janosch Schobin
}

Janosch Schobin studierte Soziologie, Mathematik und Hispanistik an der Universität Kassel. Als wissenschaftlicher Mitarbeiter am Hamburger Institut für Sozialforschung führte er das Forschungsprojekt »Freundschaft und Fürsorge « durch. Neuerdings arbeitet er über »Gesellschaftliche Dynamiken der Einsamkeit «.

E-Mail Janosch.Schobin@his-online.de
Die Zahl alleinlebender Menschen wächst. Viele von ihnen werden sich im Alter nicht auf Angehörige verlassen können, weil es diese nicht gibt. Der Aufbau und die Pflege eines Freundeskreises scheint einen Ausweg zu zeigen. Aber auch hier zeigt sich, dass die fundamentale Voraussetzung für ein Leben im Kreis der Freunde bis auf weiteres die Fähigkeit zur Selbstsorge bleiben wird. Verliert eine Person diese, sind die organisierten Akteure der öffentlichen und privaten Fürsorge gefragt. Dennoch können Freundschaften zu einem würdigen und weitgehend selbstbestimmten Leben im Alter beitragen.

Unsere Gesellschaft überaltert, die Verwandtschaftssysteme brechen weg und der Sozialstaat ist bereits heute mit seinen Aufgaben überlastet. Der Pflegenotstand droht; die Frage nach Alternativen zu institutionellen Lösungen wie etwa dem Altersheim wird immer dringlicher. Ein dritter Weg der Fürsorge jenseits von $\mathrm{Fa}$ milie und Sozialstaat bietet sich an: Die Alten könnten sich untereinander pflegen. Aber warum sollten sie das tun? Weil sie befreundet sind!?

Körperliche Fürsorge durch nahe Personen ist in der Bundesrepublik noch immer Sache der Familie oder des Partners. Allen Alarmrufen zum Trotz wird der Großteil der Pflegearbeit vor allem durch Angehörige in und außer Hauses bewältigt. Die familiären Solidaritäten sind stabil und die Ehen wie auch die eheähnlichen Lebensformen noch immer belastbar.

Aber ist deshalb bereits Entwarnung zu geben? Immerhin stagniert der Zuwachs der Scheidungsquoten und auch die Talsohle der Fertilitätsrate scheint erreicht. Ein Strukturwandel hat sich vollzogen: Aus der neolokalen Kernfamilie der Wirtschaftswunderzeit sind die vielfältigen Spielarten der multilokalen Mehrgenerationenfamilie hervorgegangen. Diese neue Klasse von Familienformen bietet schlüssige Muster der Fürsorge. Sie entlastet die
Eltern, indem sie die Großeltern für die Erziehung der Kinder mobilisiert und stützt die Großeltern, wenn diese nicht mehr für sich selbst sorgen können. (1) Eine Phase der Stabilität der Familie steht an. Mit dem absoluten Kollaps der Fürsorge durch Nahpersonen ist demnach nicht zu rechnen.

Dennoch kann keine Entwarnung gegeben werden. Es gibt mittlerweile eine zwar minoritäre aber durchaus bedeutende Restpopulation ohne familien- oder partnerzentrierte Lebensformen. Im Jahr 2000 lebten circa 17 Prozent der 40- bis 55-Jährigen außerhalb einer Paarbeziehung, wie das Deutsche Jugend Institut für seinen Familiensurvey herausfand. Etwa acht Prozent der selben Befragten hatten weder Partner noch Kinder. Für diese Gruppe gelten die Zwänge des Faktischen: Sie werden sich im Alter nicht auf Menschen verlassen können - weil es sie nicht gibt.

\section{Die Utopie: Freundschaft als Alternative}

Des Weiteren besteht kein Anlass zu der Hoffnung, dass die Population der Menschen außerhalb von Partnerschaften und Familien in den nächsten Dekaden abnimmt. Es gibt eine Art "SperrklinkenEffekt " der sich als resistent erweisen dürfte: Wer mit Ende 30 keine Familie gegründet hat, dem gelingt es auch danach meist nicht mehr. Nebst individuellen psychologischen Gründen sind für das regelmäßige Ausbleiben der Familiengründung gesellschaftliche Ursachen angebbar: Die langen Ausbildungsphasen, die Flexibilisierung der Arbeitsmärkte und die hohen Mobilitätsanforderungen tragen dazu bei, dass die Familiengründung aufgeschoben wird. (2) Am Ende ist es dann oft zu spät - das Rad der Zeit lässt sich nicht mehr zurückdrehen.

Unsere Gesellschaft produziert demnach regelhaft ein beständiges, identifi- 
zierbares Bevölkerungssegment, dessen Integranten sich nicht auf Familie oder Partner verlassen können. Es liegt nahe, hier sogleich den Staat in der Pflicht zu sehen. Die Aussicht im hohen Alter kaserniert oder von bezahlten Fremden versorgt zu werden, dürfte jedoch nur die wenigsten Menschen begeistern.

Die wechselseitige Pflege unter Freunden ist eine naheliegende Alternative zu institutionellen Patentrezepten: Die alleinstehenden Alten pflegen sich untereinander. Das wäre ganz im Sinne des Subsidiaritätsprinzips. Mit der Sozialform der Freundschaft verbindet sich heute das Versprechen auf eine allzugängliche, stets vorhandene affektive Nahbeziehung, in der sich jede Art von Fürsorge verabreden lässt, so man es wünscht.

Die Antwort wirkt auf den ersten Blick schlagend. Sie verbindet eine Sozialform zwischenmenschlicher Wärme mit den Erfordernissen und Ärgernissen des hohen Alters. Aber ist es wirklich alles so einfach mit den Freunden? Was zeigt sich, wenn man die Wunschbilder beiseite schiebt und auf die tatsächliche fürsorgliche Praxis in Freundschaften blickt?

\section{Die Realität: Hilfe und Pflege in Freundschaften}

Die empirischen Beobachtungen geben einigen Anlass zu Skepsis, wenn es um regelmäßige Hilfeleistungen und besonders, wenn es um die Leibesfürsorge unter Freunden geht. Vielerlei praktische Unterstützungsleistungen sind in Freundschaften üblich und weit verbreitet: Sei es bei Umzügen und Reparaturen, bei der Kindererziehung oder bei Notfällen, unter Freunden lassen sich - trotz einiger benennbarer Komplikationen - eine Vielzahl von Dienstleistungen verabreden. Erschwert werden regelmäßige Dienste und Hilfen unter Freunde durch drei soziale Tatsachen:

- Freundschaftsnetzwerke haben keine organisatorische Grenze. Freundeskreise decken sich selbst zwischen besten Freunden meist nur teilweise. Das hat zur Konsequenz, dass ständig Dritte, zu denen man keine Beziehung hat, die Möglichkeit tangieren, die eigenen Bedürfnisse zu organisieren.

- Die fürsorglichen Praktiken in Freundschaften sind zumeist multifunktional.Was dem einen Hilfe bei einer Reparatur ist, ist dem Anderen gleichzeitig Ablenkung und Aufheiterung. Die Abgrenzung von verrechenbarem Dienst und gemeinsamer Aktion sind meist weichgezeichnet. Das führt zu einem fundamentalen Zuschreibungsproblem: Woher weiß man, wann man eine Dienstleistung erhalten oder geleistet hat, für die man etwas entgegnen oder etwas fordern kann oder muss?

- Freundschaften sind frei von Sanktionsinstanzen. Sie geben kaum ein anderes Sanktionsmittel an die Hand um

\section{Hamburger Institut für Sozialforschung}

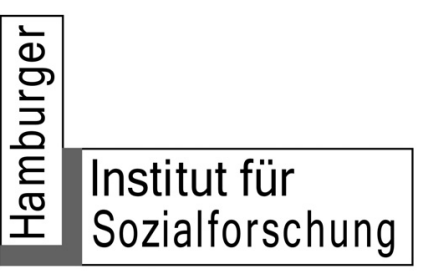

Das 1984 gegründete Hamburger Institut für Sozialforschung (HIS) ist eine unabhängige sozialwissenschaftliche und zeitgeschichtliche Forschungsstätte. Es wird von einer Stiftung getragen, deren Vorstand Jan Philipp Reemtsma ist. Das Institut hat empirische und theoretische, zeithistorische und soziologische, international ansetzende Forschungsschwerpunkte. In drei Arbeitsbereichen unter der Leitung von Ulrich Bielefeld, Heinz Bude und Bernd Greiner geht es um die bundesdeutsche Gesellschaft, um Protestbewegungen seit dem Nationalsozialismus, um Gewalt (Genozide, Terror und Krieg im 20. und 21. Jahrhundert) und Fragen und Ideen der politischen Vergesellschaftung sowie den Wandel gesellschaftlicher Normbildung und der staatlichen Sozialpolitik. Seit Ende der 1990er Jahre forscht das Hamburger Institut für Sozialforschung u. a. zu Fragen der Exklusion, Prekarität und Wohlfahrtstaatlichkeit. Zu diesen Themen fand eine Vielzahl von Tagungen statt.

Hamburger Institut für Sozialforschung, Mittelweg 36, 20148 Hamburg, Telefon 040 414097-0, E-Mail HIS@his-online.de, Internet http://www.his-online.de Quelle: Internet http://de.wikipedia.org enttäuschte Hilfserwartungen zu ahnden, als die Möglichkeit, die eigene Hilfsbereitschaft zu verringern. Selbstverstärkende Teufelskreise wechselseitiger Enttäuschung sind vorprogrammiert.

Bewältigt werden die besagten Erschwernisse durch verschiedene Strategien. So äußerten meine Gesprächspartner in qualitativen Interviews zum Beispiel oft die Ansicht, man solle seinen Freunden gegenüber Nachsicht zeigen. Wenn einer unpässlich sei, könne man sich ja auch an einen anderen wenden. Demnach bewältigen sie die Komplikationen durch Fehlerfreundlichkeit und Redundanzen. Regelmäßige praktische Unterstützung durch Freunde ist - wenn auch komplizierter als in Familien - also durchaus möglich und üblich.

Was man indes nahezu vergeblich suchen wird, sind Freunde, die einander pflegen. Das Sozioökonomische Panel (SOEP) befragte im Jahre 2008 genau 11.058 Haushalte, ob jemand im Haushalt pflegebedürftig sei. Das war 433-mal der Fall. Nur in sieben dieser Fällen wurde die Pflege ausschließlich von Freunden geleistet, in fünf Fällen erhielten sie dafür eine Bezahlung. Auf den ersten Blick ist dieser niederschmetternde Befund nicht weiter verwunderlich. Zum einen haben wir für die leibesnahe Pflege besondere Berufsstände, die darauf spezialisiert sind, den bedürftigen Körpern zu begegnen; zum anderen häuft sich der Bedarf nach leibesnahen Interventionen statistisch erst im hohen Alter. Die Gelegenheitsstruktur erzeugt einfach selten die Option, die den Akteuren die Möglichkeit geben würde, ein bestimmtes Verhalten zu zeigen.

Dennoch gibt es gute Gründe, tatsächlich eine Blockade zu vermuten, wenn es um die Pflege durch Freunde geht. In meinen Interviews stellte ich immer folgende Frage: »Wem würden Sie erzählen, wenn Sie schwer, wenn sie lebensbedrohlich krank wären? « Die Idee war, ein Gespräch darüber in Gang zu bringen, wie sich die Interviewten der Aussicht stellen, auf absehbare Zeit - und vielleicht auf Dauer - nicht für sich selbst sorgen zu können.

Die Auswertung der Antworten förderte folgenden Sachverhalt zutage: Eine Situation, in der sie nicht für sich selbst sorgen können, zogen die meisten Befragten nicht einmal gedankenexperimentell in Betracht. Viele wollten lieber sterben und 
sprachen über die Möglichkeit des Freitods. Andere planten die Veränderung der physischen Gegebenheiten ihres Wohnumfeldes. Die Generationen, die jetzt altern und sterben, sind mit markanten Autonomiebedürfnissen ausgestattet - und das hat Konsequenzen für das Erleben leiblicher Pflege durch Angehörige und Freunde: Bei der Bewältigung elementarer Lebensvollzüge von Dritten abzuhängen, widerspricht dem Selbstverständnis selbstbestimmter Existenzen zu tiefst. kungen - ein würdiges und weitestgehend selbstbestimmtes Leben im Alter. Aus menschlichen Gründen ist sie allemal der Aufbewahrung in einer totalen Institution wie einem Altersheim vorzuziehen. Die Fürsorge unter Freunden bedarf jedoch der Stützung auf drei Ebenen:

- Räumliche Arrangements, die ein gemeinsam-getrenntes Leben ermöglichen: Eines der konstitutiven Probleme bei der Organisation von Hilfe im Freundeskreis sind die Unabkömmlichkeiten, die durch eine getrennte Le-

\section{"Sperrklinken-Effekt: Wer bis Ende 30 keine Familie gegründet hat, macht es auch später nicht mehr «}

Man kann in diesem Zusammenhang folgende These wagen: Je stärker die Beziehung von Ego zu Alter Reziprozitätserwartungen durchwirken und je höher das Autonomiebedürfnis von Ego ist, umso schlechter wird Ego die Leibesfürsorge durch Alter ertragen. Freunde dürften daher mit die schlechtesten Kandidaten für die körperliche Pflege der nun sterbenden Generationen sein.

\section{Plädoyer für die gestützte Freundschaft}

Ist also die Alternative der Fürsorge unter Freunden illusorisch? Ja und nein! Möglich und üblich sind schon heute freundschaftszentrierte Lebensformen, die durch multiple Redundanzen praktische Unterstützungsleistungen, emotionale Geborgenheit und ein bestimmtes Maß an finanzieller Abstützung ermöglichen.

Die fundamentale Voraussetzung für ein solches Leben im Kreis der Freunde wird aber bis auf weiteres die Fähigkeit zur Selbstsorge bleiben. Verliert eine Person diese, sind die organisierten Akteure der öffentlichen und privaten Fürsorge gefragt. Man sollte die Freundschaft als fürsorgliche Lebensform aber deshalb nicht gleich verabschieden. Sie ermöglicht - auch unter den genannten Einschrän- bensführung entstehen. Konzepte wie die Alters-Wohngemeinschaft setzen hier an. Die Aufgabe des gewohnten Wohnumfeldes fällt Menschen im hohen Alter jedoch schwer. Der frühe Übergang in ein Wohnumfeld, das die Unterstützung durch Freunde ermöglicht, ist daher zu begrüßen. Hierzu bedarf es jedoch neuer Raumkonzepte. Kaum eine der heute üblichen Bauformen erlaubt die Mischung aus Autonomie und wechselseitiger Zugänglichkeit, nach der ein selbstbestimmtes und zugleich geborgenes Leben verlangt.

- Professionelle Unterstützung bei körperlicher Pflegebedürftigkeit: Körperliche Pflege wird - wenn sich die geschilderten Bedingungen nicht ändern - unter Freunden eine große Ausnahme bleiben. Menschen in freundschaftszentrierten Lebensformen muss in dieser Hinsicht unter die Arme gegriffen werden. Auffällig ist auch, dass Freunde meist nur dann pflegen, wenn sie dafür bezahlt werden. Möglicherweise würde eine attraktivere Gestaltung des Pflegegeldes für selbst beschaffte Pflegehilfen einen Anreiz geben sich durch Freunde pflegen zu lassen, weil man ihnen dann für ihre Pflege etwas Gleichwertiges zurückgeben könnte.

- Öffentliche Anerkennung der fürsorglichen Funktion der Freundschaft: Bis- her ist die fürsorgliche Freundschaft mehr ein Wunschbild als eine Realität. Die Freundschaft wird mit Erwartungen beladen und so implizit mit stillen Pflichten versehen - diesen entsprechen aber keinerlei Rechte. Wer einen Freund oder eine Freundin im Alter pflegt, der kann sich am Ende auf nichts berufen. Weder kann er oder sie das Erbe antreten noch eine Rente verlangen.

Wenn die Fürsorge unter Freunden eine Zukunft haben soll, müssen spezielle Rechtsvehikel entstehen, die diejenigen absichern, die Opfer für ihre Freunde erbringen.

\section{Anmerkungen}

(1) Vgl. Bien, Walther: Die multilokale Familie. Beziehungen zwischen den Generationen am Beispiel von Deutschland, in: Gutschner, Peter/Ehmer, Josef (Hg.): Das Alter im Spiel der Generationen, Wien 2000, S.193209.

(2) Vgl. Schneider, Norbert F./Ruppenthal, Silvia/ Lück, Detlef: Beruf, Mobilität und Familie, in: Zeitschrift für Familienforschung, Sonderheft, Opladen 2009,S.111-136. 